\title{
Corrigendum: Manipulating surface reactions in lithium-sulphur batteries using hybrid anode structures
}

Cheng Huang, Jie Xiao, Yuyan Shao, Jianming Zheng, Wendy D. Bennett, Dongping Lu, Laxmikant V. Saraf, Mark Engelhard, Liwen Ji, Jiguang Zhang, Xiaolin Li, Gordon L. Graff \& Jun Liu

Nature Communications 5:3015 doi: 10.1038/ncomms4015 (2014); Published 9 Jan 2014; Updated 20 Feb 2014

The original version of this Article contained an error in the order in which the first and last name of the author Laxmikant V. Saraf were displayed. This has now been corrected in both the PDF and HTML versions of the Article. 